\title{
Correction to: Modeling Disease Progression in Retinal OCTs with Longitudinal Self-supervised Learning
}

Antoine Rivail, Ursula Schmidt-Erfurth, Wolf-Dieter Vogl, Sebastian M. Waldstein, Sophie Riedl, Christoph Grechenig, Zhichao $\mathrm{Wu}$, and Hrvoje Bogunovic

\section{Correction to: \\ Chapter "Modeling Disease Progression in Retinal OCTs with Longitudinal Self-supervised Learning" in: I. Rekik et al. (Eds.): Predictive Intelligence in Medicine, LNCS 11843, https://doi.org/10.1007/978-3-030-32281-6_5}

The original version of this chapter contained an error in the third author's name. The spelling of Wolf-Dieter Vogl's name was incorrect in the header of the paper. The author's name has been corrected. 